But the determinant of the former is

$$
\mid \begin{array}{llll}
x_{1}^{\prime \prime} & 0 & 0 & 1 \\
0 & y_{2}^{\prime} & 0 & 1 \\
0 & 0 & z_{3}^{\prime} & 1 \\
0 & 0 & 0 & i
\end{array}=+x_{1}^{\prime \prime} y_{2}^{\prime} z_{3}^{\prime}
$$

and its volume is obviously equal to $x_{1}^{\prime \prime} y_{2}^{\prime} z_{3}^{\prime}$ times that of a "unit tetrahedron" $\mathrm{ABCO}$, when $\mathrm{OA}$ is measured along $\mathrm{OX}, \mathrm{OB}$ along $\mathrm{OY}$, and $\mathrm{OC}$ along $\mathrm{OZ}$, each of unit length.

Hence $\left|\begin{array}{llll}x_{1} & y_{1} & z_{1} & 1 \\ x_{2} & y_{2} & z_{2} & 1 \\ x_{3} & y_{3} & z_{3} & 1 \\ x_{0} & y_{0} & z_{0} & 1\end{array}\right|$ gives the ratio of the volume of $P_{1} P_{2} P_{3} P_{0}$ to that of the same "unit tetrahedron" in magnitude and sense.

This reasoning can be applied also in two dimensions to the area of a triangle, and to the content of a simplex in $n$ dimensions.

Remark. - The number of steps required in transforming the tetrahedron can be reduced to three, and Theorem II. is not required if we start with $P_{0}$ coincident with $O$.

R. F. Muirhead.

A Note on Induction.-An application of the method of Induction to the proof of a general result or theorem should involve two steps: (i) the discovery of the result to be proved by the consideration of particular cases ; (ii) a proof, by Mathematical Induction, or otherwise, that the result, found to be true in particular cases, is universally true.

The first step is often omitted, the pupil being supplied with the result and asked to show that it is universally true. A most valuable part of the work is thereby lost; to find a formula from the examination of particular cases demands careful observation and a power of generalising which should be cultivated. In fact, step (i) by itself, apart from step (ii), is an excelient exercise. It is, of course, in many cases, extremely difficult, if not impossible, for 
the beginner to see a general formula which will include all the particular results obtained; the object of this note is simply to emphasise the value of the exercise when it is not too difficult.

(1) A very simple example is the finding of the sum of the first $n$ odd integers.

(i) We have

$$
\begin{aligned}
1 & =1, \\
1+3 & =4, \\
1+3+5 & =9, \\
1+3+5+7 & =16 ;
\end{aligned}
$$

going no further, we observe that the sums obtained are squares, that the sum of two terms is the square of 2 , the sum of three terms is the square of 3 , the sum of four terms is the square of 4 ; these results suggest that " the sum of the first $n$ odd integers is $n^{2}$."

(ii) We now show by Mathematical Induction, or otherwise, that this statement is true for all values of $n$.

(2) Consider the series

$$
0,1,1,2,3,5,8,13,21, \ldots
$$

(i) We observe that any term, after the second, equals the sum of the two preceding terms; taking this as the law of the series we shall have $\mathrm{T}_{n}=\mathrm{T}_{n-1}+\mathrm{T}_{n-2}$.

To get some information about the sum of $n$ terms of the series, which we shall denote by $S_{m}$, let us write $S_{1}$ below $T_{1}, S_{2}$ below $T_{2}$, $\mathrm{S}_{3}$ below $\mathrm{T}_{3}$, etc.

$$
\begin{aligned}
& 0,1,1,2,3,5,8,13,21, \ldots . \\
& 0,1,2,4,7,12,20,33,54, \ldots .
\end{aligned}
$$

An observant pupil will suggest that $S_{n}=T_{n+2}-1$; we see that this is true for $n=1,2,3,4, \ldots .7$.

(ii) We now examine, by Mathematical Induction, if the formula suggested holds for all values of $n$.

(3) A very good example is the following:-Find the sum of $n$ terms of the series $\frac{1}{1 \cdot \frac{4}{4}}, \frac{1}{4 \cdot 7}, \frac{1}{7 \cdot 10}, \frac{1}{10 \cdot \overline{1} 3}, \ldots$.

Assuming the law of the series to be that the first factors of the denominators are in A.P., and that the second factor of any denominator exceeds the first by 3 , we find $T_{n}$ to be $\frac{1}{(3 n-2)(3 n+1)}$. 


\section{A NOTE ON INDUCTION.}

We have also

$$
\begin{aligned}
& S_{1}=\frac{1}{4}, \\
& S_{2}=\frac{1}{4}+\frac{1}{4 \cdot 7}=\frac{2}{7}, \\
& S_{3}=\frac{2}{7}+\frac{1}{7 \cdot 10}=\frac{8}{10} ;
\end{aligned}
$$

going no further, we observe that the numerators of $S_{1}, S_{2}, S_{3}$ are $1,2,3$, the denominators are $4,7,10$, i.e., $3.1+1,3.2+1$, $3.3+1$; so that the results obtained suggest

$$
\mathrm{S}_{n}=\frac{n}{3 n+1} \text {. }
$$

(4) A very good series for simple inductive work is the series $1,3,7,15,31, \ldots$ By observation we get

$$
\begin{aligned}
& \mathrm{T}_{n}=2^{n}-1, \\
& \mathrm{~T}_{n}=2 \mathrm{~T}_{n-1}+1, \\
& \mathrm{~S}_{n}=\mathrm{S}_{n-1}+n, \text { etc. }
\end{aligned}
$$

The $n^{\text {th }}$ term of this series is, by the way, the solution to the old problem: "A market woman sells to a first person the half of her apples and half an apple more; to a second, the half of the remainder and half an apple more; after $n$ transactions of this kind all her apples are sold. How many had she?"

(5) Some interesting examples can be obtained from Pascal's triangle of numbers. The first number in each row of the triangle is 1 , and any other number in a particular row is the sum of the number immediately above it and the number which precedes the latter in the same row :

$\begin{array}{rrrrrrr}1 & & & & & \\ 1 & 1 & & & & \\ 1 & 2 & 1 & & & \\ 1 & 3 & 3 & 1 & & \\ 1 & 4 & 6 & 4 & 1 & \\ 1 & 5 & 10 & 10 & 5 & 1\end{array}$

We observe that in any row (so far as they have been written down) numbers equidistant from the ends of the row are equal. Is this true for the $n^{\text {th }}$ row? The summation of the numbers in the successive rows suggests that the sum of the numbers in the $n^{\text {th }}$ row is $2^{n-1}$; is this true? We observe also that if we add the numbers in a line parallel to the hypotenuse, starting from one of the l's on the left, the sum obtained is the number immediately below the last number included in the sum. And so on. 